\title{
NOISE FILTRATION OF SHOCKWAVE PROPAGATION IN MULTI-LAYERED SOILS
}

\author{
LAITH I. NAMIQ \& YOUSOF Q. ABDALJALIL \\ Retired Professor, Geotechnical Department, AKRF, Inc., USA.
}

\begin{abstract}
Numerical methods, and especially the finite-element method (FEM), are usually adopted for the analyses of shockwave propagation in nonlinear inelastic media. Noise or spurious oscillations, in the calculated stresses and displacements, frequently appear in the FEM solutions. This article introduces and describes a numeric filter based on least-square analysis that can smooth out such fictitious noise. The sliced least-square method (SLSM) filter is implemented in a finite elements program that solves 1D time integration of dynamic equilibrium sets of equations that simulate shockwave propagation in multi-layered soils supported by a hard stratum. Elastic and elasto-viscoplastic material models with dynamic yield surface constitutive relations are invoked to model sand, clay, and concrete materials in the analyses. Results of the analyses of shockwave propagation in layers of soil and concrete without the filter are compared with identical conditions with the inclusion of the new filter in the finite-element program. Oscillations in calculated stresses and displacements were observed in the results when no filter was included in the solution program. Solution results showed little or no noise with the application of the new filter. The predicted FEM analyses results were compared with physical test results with very good to excellent comparisons obtained.

Keywords: elasto-viscoplastic material model, finite elements, implicit time integration, spatial filter, spurious oscillations, wave propagation.
\end{abstract}

\section{INTRODUCTION}

Analyses of wave propagation in multi-layered soil profiles are required in many geotechnical engineering problems. Among these problems is prediction of magnitudes and frequencies of shock-generated body and surface waves at the location of nearby structures. Closed-form solutions do exist, and utilize assumptions of simple boundary conditions and elastic or simplified elastic-plastic soil behaviour. However, closed-form solutions become intractable if more representative constitutive relations for earth materials are utilized, and if large strains and/or cycles of loading and unloading are to be accounted for. Hence, numerical methods, and especially the finite-element method (FEM), are usually adopted for the analysis of these and similar problems. The problem is that noise or spurious oscillations in the calculated stresses and displacements frequently appear in the FEM solutions. Calculations with such imprecision have been recognized, and attempts to circumvent these spurious oscillations and improve upon the solutions have been and are being carried out in several ways. Modifications to the time integration operator adopted in the FEM solution technique were proposed[1]. The incorporation of additional sinusoidal terms in the solution method was attempted [2]. A cut-off wave length was suggested for any given FEM mesh [3]. The addition of harmonic functions to the conventional interpolation function to enrich the FEM formulation was introduced [4]. Calculations of critical element size to be avoided in FEM mesh construction were explored [5]. The basic idea in all these attempts is the modification of one or more phase(s) of the solution technique, i.e. interpolation function, element size, integration scheme, etc. To summarize, all proposed solutions dealt with a pre-result output. A different approach was to improve upon the FEM post-result solution by use of digital [6] or spatial filters [7]. A simple, efficient, new numerical spatial filter that smoothens out FEM-generated fictitious noise is introduced and detailed herein. 


\section{SLICED LEAST-SQUARE METHOD FILTER (SLSM)}

Least-square analysis forms the mathematical basis of the introduced filter. The least-square method can be expressed mathematically as follows: Let $\psi(x)$ be defined on the interval $a \leq x \leq b$ and let $\varphi\left(x, C_{1}, C_{2}, \ldots, C_{n}\right)$ be the function that is to approximate $\psi$ over the interval, given the selection of values for the parameters $C_{i}, i=1,2, \ldots, n$. The basic concept is that the weighted squared error over the whole interval should be a minimum. The error $\varepsilon$ at a given point in the above-defined interval is given by eqn (1):

$$
\varepsilon=\psi(x)-\phi\left(x, C_{1}, C_{2}, \ldots, C_{n}\right)
$$

and the integral, $\Gamma_{2}$ in eqn (2), is to be minimized:

$$
\Gamma_{2}\left(C_{1}, C_{2}, \ldots, C_{n}\right)=\int_{a}^{b} w(x)\left\{\psi(x)-\phi\left(x, C_{1}, C_{2}, \ldots, C_{n}\right)\right\} \cdot d x
$$

where $w(x)$ is a weighting function. The target function, $\psi$, is available at a given number of points, $\mathrm{m}$. Thus, the least-square error procedure is defined as follows:

$$
\begin{aligned}
& C_{1} \sum_{k=1}^{m} w\left(x_{k}\right) \cdot \phi_{i}\left(x_{k}\right) \cdot \phi_{1}\left(x_{k}\right)+\ldots+ \\
& C_{i} \sum_{k=1}^{m} w\left(x_{k}\right) \cdot\left(\phi_{i}\left(x_{k}\right)\right)^{2}+\ldots+ \\
& C_{n} \sum_{k=1}^{m} w\left(x_{k}\right) \cdot \phi_{i}\left(x_{k}\right) \cdot \phi_{n}\left(x_{k}\right)=\sum_{k=1}^{m} w\left(x_{k}\right) \cdot \phi_{i}\left(x_{k}\right) \cdot \psi\left(x_{k}\right) .
\end{aligned}
$$

Defining the elements of the matrix $\mathbf{S}$ and vector $\mathbf{T}$ as follows:

$$
\begin{gathered}
S_{i, j}=\sum_{k=1}^{m} w\left(x_{k}\right) \cdot \phi_{i}\left(x_{k}\right) \cdot \phi_{j}\left(x_{k}\right) \\
T_{i}=\sum_{k=1}^{m} w\left(x_{k}\right) \cdot \phi_{i}\left(x_{k}\right) \cdot \psi\left(x_{k}\right)
\end{gathered}
$$

one can write $n$ equations, where $\mathrm{n}$ is the order of the polynomial fit plus one, in matrix notation as in eqn (6):

$$
S \cdot C=T
$$

where $\mathbf{C}$ is a vector containing e coefficients $C_{1}, C_{2}, \ldots, C_{n}$. Solving eqn (6) yields the values of $C_{1}$ through $C_{n}$. And then the approximating function, $\phi(x)$, can be computed, at any point in the interval $(a, b)$, using eqn (7):

$$
\phi(x)=\sum_{i=1}^{n} C_{i} \cdot x^{i-1}
$$

The weight function, $\mathrm{w}\left(\mathrm{x}_{\mathrm{k}}\right)$ is assigned a value of 1.0 in SLSM as no given Gauss integration point is believed to be more accurate than other points.

The least-square regression analysis method (LSM) may be applied to smooth FEM-calculated output (e.g., stresses) of wave propagation throughout the domain of interest of a solid body. Noise-free response is then obtained. However, illusory smoothening and flattening of sharp peaks in computed stresses, as well as erratic response ahead of the wave front, is 
produced by application of LSM to the FEM solution output. To circumvent this problem, application of LSM is preceded by an additional procedure designed to eliminate the introduction of further erratic results in the FEM output. This procedure is developed herein, and together with LSM, defines SLSM.

SLSM is achieved by sorting in linear order, in vector $\mathbf{V}$, the magnitude of generated dynamic loading stress components, at element Gauss integration points along the wave propagation axis. This is followed by searching, per a pre-set criterion, the FEM output for 'jumps' in the calculated stress' magnitudes between arranged consecutive Gauss integration points. The complete set of Gauss integration points is then divided into subsets delimited by consecutive stress 'jumps'. A slice that is the set of Gauss integration points between two 'jumps' is thus generated. LSM is subsequently operated on each slice individually. Finally, displacements, velocities, and acceleration vectors are modified according to the new stress value (i.e. after LSM application) at each Gaussian integration point.

\subsection{Stress 'jump' identification criterion}

A stress 'jump' is identified at each time step when any of the following conditions is true:

2.1.1 Condition (a)

$D<=R$ as shown in Fig. 1, other variables shown in the figure are given by eqn (8) through (11).

$$
\begin{gathered}
A_{1}=\sigma_{1} / \sigma_{\max } \\
A_{2}=\left(\left(\frac{\sigma_{2}}{\sigma_{\max }}\right)+\left(\frac{\sigma_{3}}{\sigma_{\max }}\right)\right) / 2 \\
R=\left|A_{2}-\alpha\left(A_{1}+A_{2}\right)\right|
\end{gathered}
$$

where $\alpha$ is a dimensionless factor. $\mathrm{D}$ is calculated using eqn (11):

$$
D=\left|A_{2}-\left(\sigma_{3} / \sigma_{\max }\right)\right|
$$

In Fig. 1, $\mathrm{d}$ is the distance along the selected search direction, and $\mathrm{L}$ is the cumulative length of finite elements (FE) along that search direction.

\subsubsection{Condition (b)}

$D<=R$ as shown in Fig. 2. The variables shown in Fig. 2 are given by eqn (12) through (15):

$$
\begin{gathered}
A_{1}=\left(\left(\frac{\sigma_{j-3}}{\sigma_{\max }}\right)+\frac{\sigma_{j-2}}{\sigma_{\max }}\right) / 2 \\
A_{2}=\left(\left(\frac{\sigma_{j-1}}{\sigma_{\max }}\right)+\frac{\sigma_{j}}{\sigma_{\max }}\right) / 2 \\
R=\left|A_{2}-\alpha\left(A_{1}+A_{2}\right)\right| \\
D=\left|A_{2}-\left(\sigma_{j} / \sigma_{\max }\right)\right|
\end{gathered}
$$




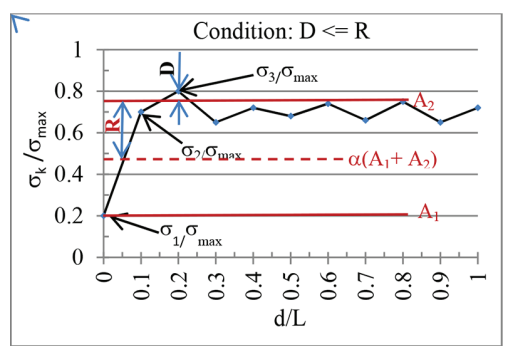

Figure 1: Condition (a) for stress 'jump' identification.

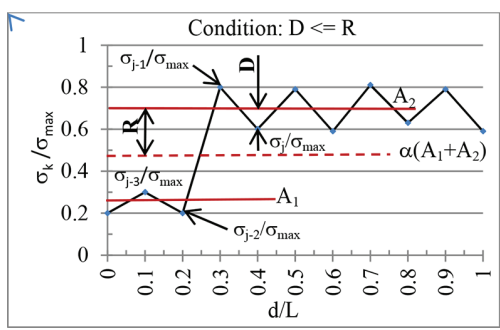

Figure 2: Condition (b) for stress "jump" identification.

\subsubsection{Condition (c)}

$D<=R$ as shown in Fig. 3, where $\mathrm{R}$ is given by eqn (16), and the rest of the variables are as defined for condition (b).

$$
R=\left|A_{2}-\left(\frac{\sigma_{j-3}}{\sigma_{\max }}\right)\right|
$$

If none of the conditions (a) through (c) are applicable, then LSM is applied to the stresses calculated at the end of current time step, Fig. 4.

\section{EXAMPLES}

\subsection{Analysis of an elastic rod}

Linear-elastic slender column was analysed under a variety of applied loadings as given by eqns (17) through (20):

(a) Initial conditions:

$$
\boldsymbol{U}_{o}=\dot{\boldsymbol{U}}_{o}=0
$$

where $\boldsymbol{U}$ is the vector of nodal displacements in the z-direction. Vectors and matrices are in bold type. Note that ' $u$ ' is the displacement in the z-direction and ' $\mathrm{v}$ ' is the displacement in the radial direction; see Fig. 5.

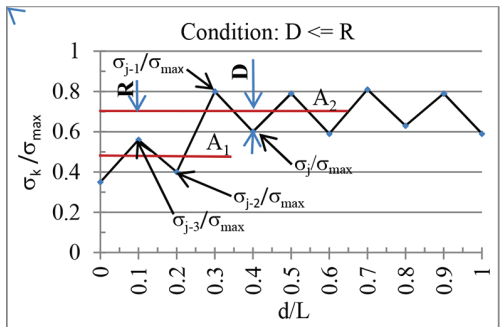

Figure 3: Condition (c) for stress "jump" identification.

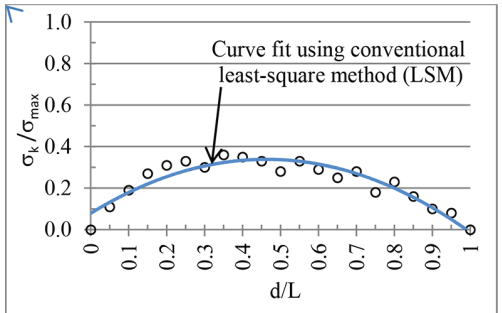

Figure 4: Curve fit where no stress "jump" is identified. 


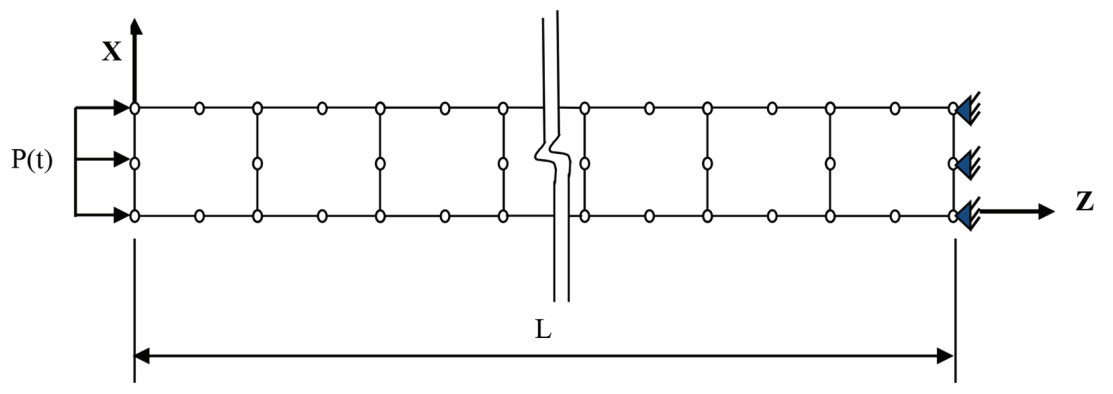

Figure 5: Finite element mesh for slender rod. All side nodes are restrained in the X-direction.

(b) Boundary conditions:

$$
\begin{gathered}
v=0 ; z=[0, L] \\
\boldsymbol{u}=0 ; z=L
\end{gathered}
$$

(c) Applied load:

$$
P(t)=\left\{\begin{array}{c}
0, t \leq 0 \\
2070, t=0.006 s \\
<2070, t>0.006 s
\end{array}\right.
$$

where $\mathrm{t}$ is time in seconds, and $\mathrm{P}(\mathrm{t})$ is in $\mathrm{kPa}$. The variation of applied pressure with time is given in [9].

The pressure $\mathrm{P}(\mathrm{t})$ is uniformly distributed on the element surface where $\mathrm{z}=0$. Axisymmetric 8-node isoparametric elements were used in that analysis. A cubic polynomial fit in the SLSM was implemented, eqn (21):

$$
\sigma(z)=A+B \cdot z+\mathrm{C} \cdot \mathrm{z}^{2}+\mathrm{D} \cdot \mathrm{z}^{3}
$$

where A, B, C, and D are coefficients that were calculated using the standard least-square procedure. The exact and filtered solutions are shown on Fig. 6. The filtered solution (i.e. calculated axial stress versus distance along the slender rod) closely matches the exact solution.

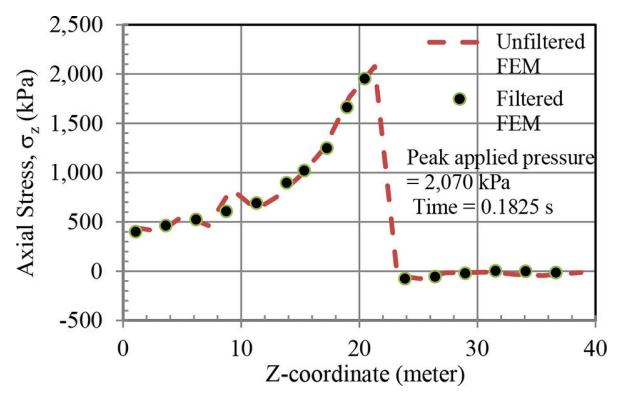

Figure 6: Elastic analysis of a slender load under dynamic load. 

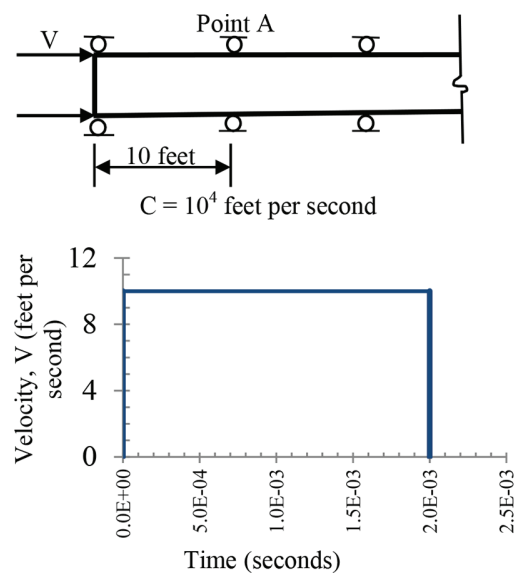

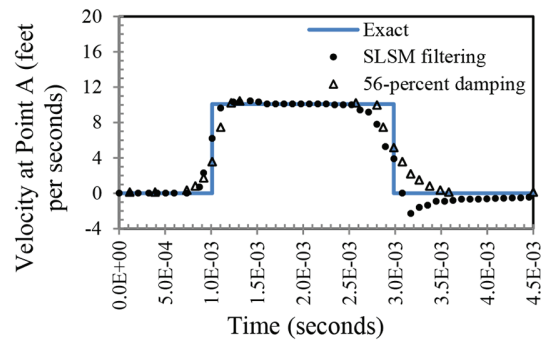

Figure 7: (a) Model of a slender rod and Variation of applied velocity versus time. (b) Comparison of the SLSM filtered solution to artificial damping method cited by Belytschko et al. [10].

The problem of a slender column under axial strep velocity applied at the free end was solved, Fig. 7a. The problem was presented in Belytschko et al. [10], where artificial damping in the FEM solution was employed. The exact solution, FEM solution with artificial damping, and the SLSM filtered FEM solution, are shown in Fig. 7b. As can be seen, the SLSM solution is close to the exact one.

\subsection{Non-linear analysis of a soil column}

The problem of a soil column subjected to time-varying, vertical pressure was solved. The material model is an elasto-viscoplastic modified Cam clay model [11]. Configuration of FE and boundary conditions are the same as for the case of elastic rod (Section 3.1). The soil column consists of normally consolidated clay with decreasing void ratio with depth. The applied load is as given by eqns (17) through (20). However, in this case, the peak applied pressure, $P_{o}$, is $207 \mathrm{kPa}$.

Attenuation factor, $\alpha$, is given by eqn (22):

$$
\alpha=\Delta \sigma_{z} / P_{o}
$$

where $\Delta \sigma_{z}$ is the change in axial stress and is calculated using the output of the FEM program. Variation of the attenuation factor with non-dimensional depth (i.e. along the axis of the soil column) is shown in Fig. 8. As a check on the validity of the solution, the calculated variation of attenuation factor (with depth) is compared to that published in [9]. The solution shown in [9] is presented as Fig. 9. As can be seen from Figs 8 and 9, the variation of the attenuation factor with depth has the same characteristics.

Change in axial (vertical) stress, using an elasto-viscoplastic material model for the clay column with peak overpressure of 2,070 kPa is calculated. Propagation of the stress wave is shown in Fig. 10. SLSM is applied to the calculated change in axial stress and one result is shown in Fig. 11. The filtered solution shows larger compressive and smaller tensile stresses compared with the unfiltered solution. 


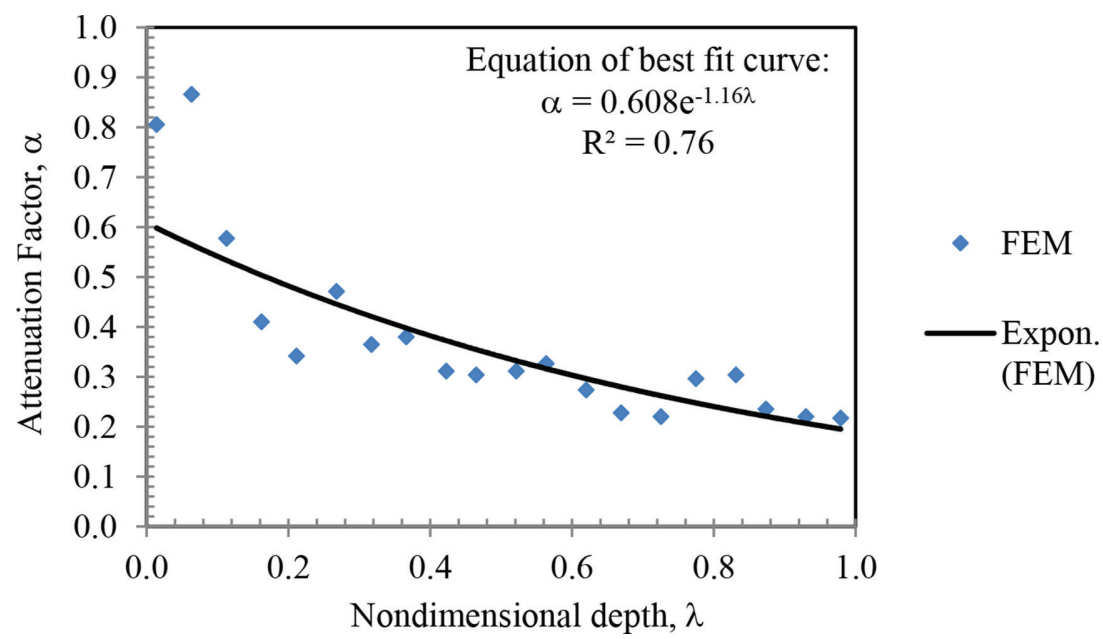

Figure 8: Variation of the attenuation factor versus non-dimensional depth.

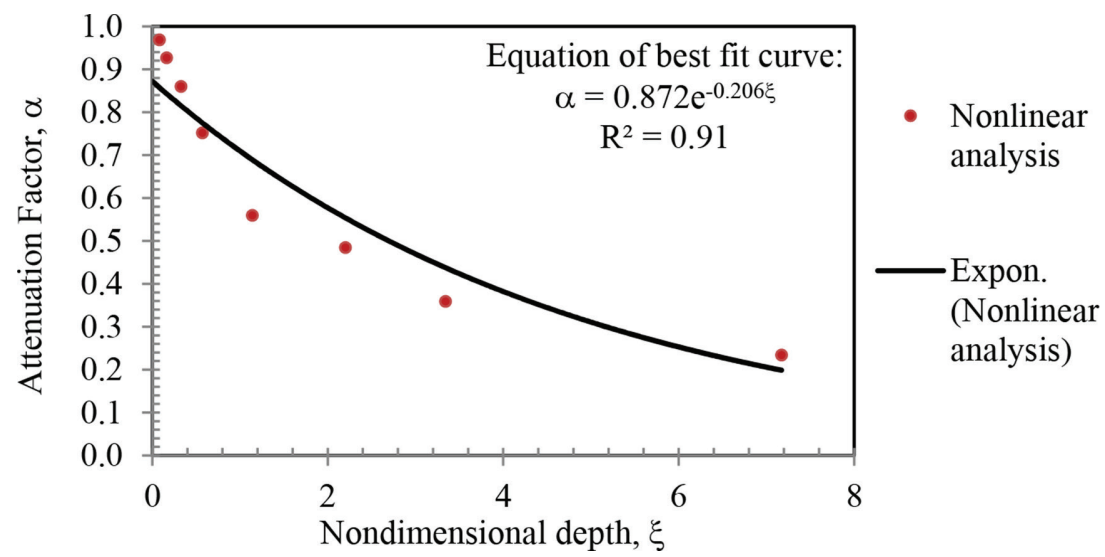

Figure 9: Variation of attenuation factor versus depth using bilinear 1-D stress-strain relationship (after Hendron and Auld [9]).

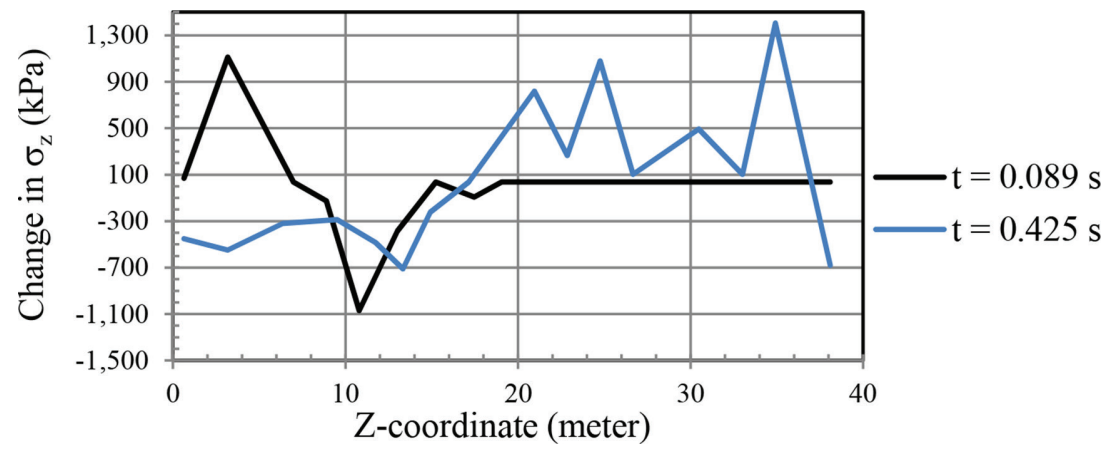

Figure 10: Stress wave propagation in a clay column using elasto-viscoplastic analysis. 


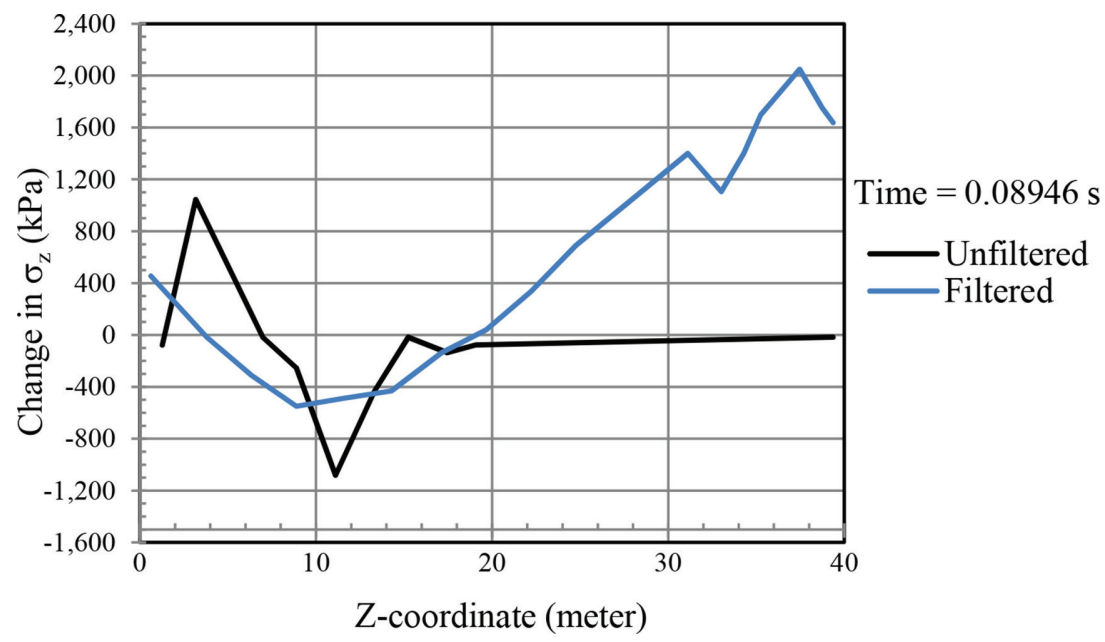

Figure 11: Effect of stress smoothening on predicated change of stress in clay column (peak overpressure $=2,070 \mathrm{kPa}$ ).

\subsection{Non-linear analysis of sand and concrete strata}

A two-strata column with plain concrete on top of sand and with an interface element between the two materials was analysed. The thickness of each stratum was $19.97 \mathrm{~m}$, and the interface element was $0.06 \mathrm{~m}$ thick. Plain concrete was modelled using a non-linear stress-strain relationship. Sand was modelled using an elasto-viscoplastic version of modified Cam clay. The applied load, at the top of the plain concrete stratum, was the same as for the clay column, as shown in Figs 10 and 11.

The change in axial stress calculated using a linear, elastic material model was compared with that using the elasto-viscoplastic material model, as shown in Fig. 12. A reduction in peak axial stress was noticed in the case of the elasto-viscoplastic material model. The reduction can be attributed to energy dissipation caused by plastic strain in the concrete and sand, given the high pressure applied with a peak of 2,070 $\mathrm{kPa}$.

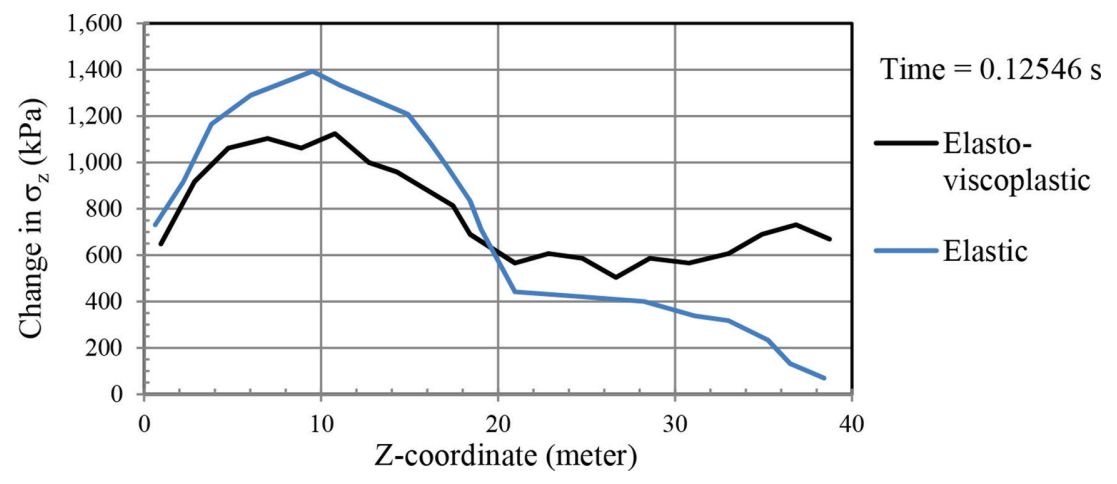

Figure 12: Effect of analysis type on predicted change in axial stress for the case of plain concrete on sand stratum. 


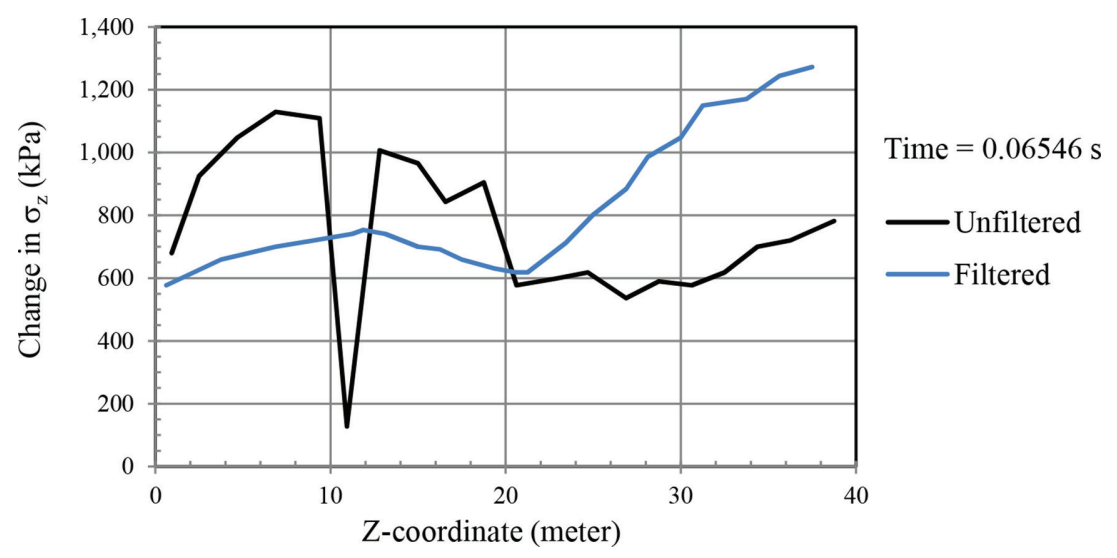

Figure 13: Effect of stress smoothening on predicted change in stress for the case of plain concrete on sand stratum (elasto-viscoplastic analysis).

An example of the output of SLSM filtered and unfiltered change in stress is shown in Fig. 13. The filtered solution indicates a smooth variation of the change in axial stress versus depth. It also indicates that a peak in stress has been reached near the fixed end of the concrete-and-sand column. The unfiltered solution indicates a tensile stress jump near a depth of $11 \mathrm{~m}$, which is near the middle of the concrete stratum. From the mechanics of stress wave propagation, such tensile stress jump cannot be explained, given that one end of the concreteand-sand column is free and the other is fixed (in the direction of wave propagation).

\section{CONCLUSIONS}

The application of the FEM to the problem of shock wave propagation in multi-layer continua, whose constitutive laws are complex, provides results that cannot be obtained using existing closed-formed solutions. Spurious oscillations in FE solutions are inherent because of the limited cut-off frequency in FE meshes. Spurious oscillations observed in the case of material non-linearity are higher than those in the elastic medium.

A spatial filter, the SLSM, based on the least-squares method, is developed herein. The SLSM filter proved effective in linear elastic analyses in reducing the magnitude of the spurious oscillations in the calculated axial stress in a slender column, while preserving the shape of the advancing stress wave.

The elasto-viscoplastic solution showed attenuation trend in the axial stress, which matches with the published data very well. The SLSM filter provides smoothening of the calculated stress in elasto-viscoplastic slender columns composed of one or more materials.

\section{REFERENCES}

[1] Belytschko, T. \& Mullen, R., Mesh partitions of explicit-implicit time integration. Formulations and Computational Algorithm in Finite Element Analysis, ed. K.J. Bathe, T. Oden \& W. Wunderlich, MIT Press: Cambridge, Massachusetts, U.S.A., pp. 672-690, 1978.

[2] Pain, H.G., The Physics of Vibrations and Waves, John Wiley and Sons Ltd.: London, 1976.

[3] Kreig, R.D. \& Key, S.W., Transient shell response by numerical time integration. Advances in Computational Methods in Structural Mechanics and Design, eds J.T. Oden, W.W. Clough \& Y. Yamamoto, UAH Press: Huntsville, Alabama, U.S.A., 1972. 
[4] Ham, S. \& Bathe, K.J., A finite element method enriched for wave propagation problems. Computers and Structures, 94-95, pp. 1-12, 2012. https://doi.org/10.1016/j.compstruc.2012.01.001

[5] Schmicker, D., Duczek, S., Liefold, S. \& Gabbert, U., Wave propagation analysis using high-order finite element methods: spurious oscillations excited by internal element eigenfrequencies. Technische Mechanik, 34(2), pp. 51-71, 2014.

[6] Okrouhlik, M., Ptak, S. \& Valdek, U., Self-assessment of finite element solutions applied to transient phenomena in solid continuum mechanics. Engineering Mechancis, 16(2), pp. 103-121, 2009.

[7] Martel, L., Love wave propagation across a step by finite elements and spatial filtering. Geophysical Journal of the Royal Astronomical Society, 61, pp. 659-677, 1980. https://doi.org/10.1111/j.1365-246X.1980.tb04837.x

[8] Cope, R.J., Sawko, F. \& Tickell, R.G., Computer Methods for Civil Engineers, McGrawHill Book Company, Ltd.: U.K., 1982.

[9] Hendron, A.J. \& Auld, H.E., The effect of soil properties on the attenuation of airblast-induced ground motions. Proceedings of Symposium on Wave Propagation, The University of New Mexico, Albuquerque, New Mexico, U.S.A., 1978.

[10] Belytschko, T., Chiapetta, R.L. \& Bartel, H.D., Efficient large scale non-linear transient analysis by finite elements. International Journal of Numerical Methods in Engineering, 10, pp. 579-596, 1976. https://doi.org/10.1002/nme.1620100308

[11] Atkinson, J.H. \& Bransby, P.L., The Mechanics of Soils: An Introduction to Critical State Soil Mechanics, McGraw-Hill Ltd.: U.K., 1978. 